\title{
Thesis
}

\section{MDCI: Model-driven continuous integration}

\author{
Vicente García-Díaz * B. Cristina Pelayo García-Bustelo and Juan Manuel Cueva Lovelle \\ Department of Computer Science, University of Oviedo, Asturias, Spain \\ E-mail: \{garciavicente,crispelayo,cueva\}@uniovi.es
}

Keywords: Model-driven engineering, continuous integration, self-adaptive, incremental change, domain-specific

On June 29, 2011, Vicente García-Díaz defended his $\mathrm{PhD}$ thesis at Oviedo University, titled: "MDCI: Model-Driven Continuous Integration". The research was funded by the Government of the Principality of Asturias (Spain). Vicente García-Díaz defended his dissertation in a publicly open presentation held in the School of Computer Engineering at Oviedo University, and was able to comment on every question raised by his thesis committee and the audience. The thesis was supervised by his advisors, Juan Manuel Cueva Lovelle and B. Cristina Pelayo García-Bustelo, and the rest of his thesis committee, Luis Joyanes Aguilar, Oscar Sanjuán Martínez, Manuel Pérez Cota, Francisco Javier Segovia Pérez and Victor Hugo Medina García. The thesis has been read and approved by his thesis committee, receiving the highest rating. All of them were present at the presentation.

\section{Thesis summary}

The purpose is to create a process in which the continuous integration practice can be applied to model-driven software developments in an effective way, through which software developments can benefit jointly and simultaneously from the improvements and advantages provided by the model-driven engineering development approach and the continuous integration practice. That helps systems to change based on environmental conditions.

\footnotetext{
*Corresponding author.
}

The model-driven engineering approach is the last step of software engineering in the search for development approaches that raise the level of abstraction to the point that experts in a domain of knowledge, outside the computer world, are able to guide and change the logic of computer systems. To that end, we proposed a simplified behavior modeling notation [1].

The continuous integration practice is a recommendation of the most widely accepted development methodologies that aims to carry out automatic software integrations in early stages of development, offering benefits such as reducing the inherent risk that every project has. We simultaneously applied both model-driven engineering and continuous integration during the development of this thesis in a real working project [2]. In it, the system could automatically adapt itself to the changing context of different manufacturing processes.

By merging the model-driven engineering and the continuous integration practice, the aim is to provide to development teams that work using some kind of model-driven engineering initiative, the possibility to integrate their developments in a continuous and distributed way. At the same time, customers, the real experts in the domain of knowledge in their field of business, can benefit from the increased level of abstraction in developing techniques. Thus, they are able to modify their own computer system without the help of external technical staff, so saving time and costs.

To meet the objectives, a prototype which saves all the current constraints that do not allow the union 


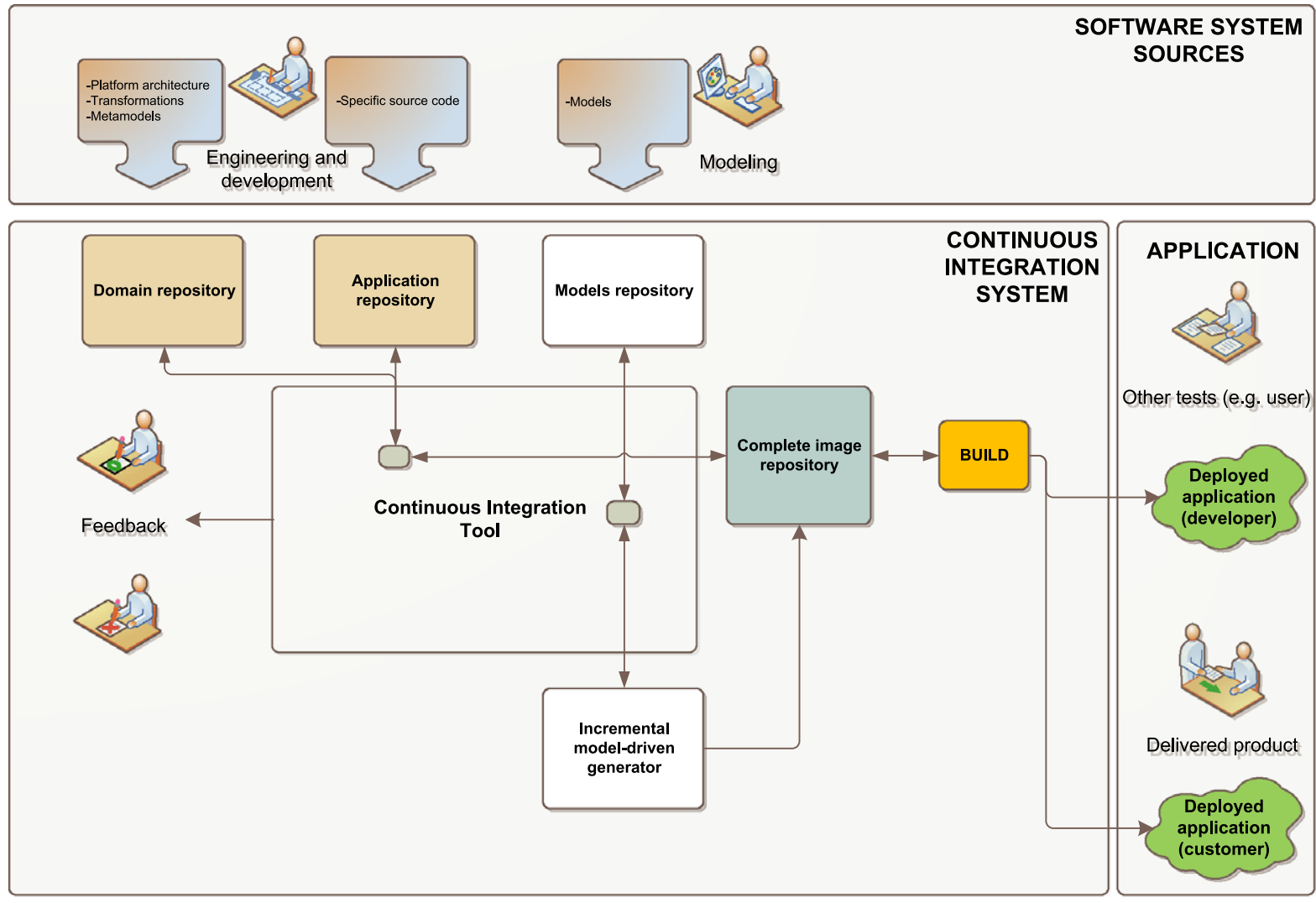

Fig. 1. MDCI Overview.

between these two recent tools of software engineering was build. Some of the main problems found were related to the selection of an appropriate development initiative [2], the quality of transformation models [3] or the correction in the detection of new models representing systems [4]. The separation of work in different blocks can provide solutions resulting in an iterative and incremental work from beginning to end. Figure 1 shows an overview of the system.

To analyze the benefits of the proposal in this work compared to other development possibilities, an evaluation was performed by creating different test cases in which the measurement of different parameters can give a numerical estimate of the real benefits obtained. The descriptive analysis, the hypothesis testing and regression techniques allow a better interpretation of results.

We are working on real cases of application in which Model-Driven Continuous Integration can provide many benefits. For instance, recommender systems applied to electronic books [5] that can be adapted to the specific characteristics of the environment, having context awareness. Another example is the generation of learning management system modules [6], being a first step towards creating educational environments adaptable to the environment. In addition, we plan to use our system to support context-awareness in a robotics framework [7].

Finally, the process was defined by answering various questions posed to facilitate its comprehension and understanding.

\section{References}

[1] H. Fernández-Fernández, E. Palacios-González, V. GarcíaDíaz, B.C. Pelayo García-Bustelo, O. Sanjuán Martínez, and J.M. Cueva Lovelle, Sbpmn - an easier business process modeling notation for business users, Computer Standards \& Interfaces 32(1-2) (2010), 18-28.

[2] V. García-Díaz, H. Fernández-Fernández, E. Palacios-González, B.C. Pelayo G-Bustelo, O. Sanjuán-Martínez, and J.M. Cueva Lovelle, Talisman MDE: Mixing MDE principles, Journal of Systems and Software 83(7) (2010), 1179-1191. 
[3] J.B. Tolosa, O. Sanjuán Martínez, V. García-Díaz, B.C. Pelayo García-Bustelo, and J.M. Cueva Lovelle, Towards the systematic measurement of atl transformation models, Software: Practice and Experience 41(7) (2011), 789-815.

[4] V. García-Díaz, B.C. Pelayo García-Bustelo, O. Sanjuán Martínez, E.R. Núñez-Valdéz, and J.M. Cueva Lovelle, Mctest: Towards an improvement of match algorithms for models, IET Software 6(2) (2012), 127-139.

[5] E.R. Núñez-Valdéz, J.M. Cueva Lovelle, O. Sanjuán Martínez, V. García-Díaz, P. Ordóñez de Pablos, and C.E. Montenegro Marín, Implicit feedback techniques on recommender systems applied to electronic books, Computers in Human Behavior 28(4) (2012), 1186-1193.

[6] C.E. Montenegro Marín, J.M. Cueva Lovelle, O. Sanjuán Martínez, and V. García-Díaz, Domain specific language for the generation of learning management systems modules, Journal of Web Enginnering 11(1) (2012), 23-50.

[7] S. Mendez Nunez, F. Ortin, M. García, and V. García-Díaz, Computational reflection in order to support context-awareness in a robotics framework, in: $S E K E$, Knowledge Systems Institute Graduate School, 2011, pp. 533-538. 